\title{
Simulation numérique de l'endommagement à l'aide du modèle Francfort-Marigo
}

\author{
Grégoire Allaire*, Sylvie Aubry ${ }^{\dagger} \&$ François Jouve ${ }^{\ddagger}$
}

\begin{abstract}
Résumé
Dans cet article, on présente la mise en oeuvre de simulations numériques de l'évolution de l'endommagement selon le modèle de Francfort et Marigo. Il s'agit d'un modèle d'endommagement partiel, quasi-statique, irréversible, d'une structure élastique dans le cadre de petites déformations. Ce modèle permet de prévoir la formation et l'évolution des zones endommagées dans la structure. Pour que ce modèle soit bien posé, il est nécessaire d'avoir recours à l'homogénéisation car des mélanges fins de matériaux sain et endommagé peuvent naturellement apparaitre. A l'aide de cas tests simples (poutre en flexion, éprouvette pré-fissurée), on montre les principaux avantages et les limitations d'une telle modélisation.
\end{abstract}

\section{Introduction}

La mécanique de l'endommagement permet de décrire la détérioration des matériaux sous l'influence de forces extérieures évolutives. La cause de cette détérioration réside dans des mécanismes micro-structuraux (du type micro-fissures ou autres) qu'on ne cherche pas à décrire précisément ici. On dit que l'endommagement est partiel lorsque le matériau endommagé possède encore une élasticité résiduelle, quoique diminuée par rapport à celle du matériau sain d'origine. L'endommagement est bien sûr irréversible car une zone endommagée ne peut plus retrouver son élasticité initiale. Il existe de très nombreux modèles d'endommagement dont nous ne discuterons pas les mérites respectifs (voir, par exemple, [5]). Nous nous contenterons ici de rappeler un modèle d'endommagement introduit par Francfort et Marigo [4] auquel nous renvoyons pour plus de détails sur son établissement. Ce modèle est quasi-statique, comme la plupart des autres modèles, c'est-à-dire que les effets d'inertie sont négligés et l'évolution temporelle se résume à une suite de régimes permanents.

Le but de cet article est de présenter une méthode numérique et des simulations numériques en 2-D pour ce modèle de Francfort et Marigo. Des calculs numériques ont déjà été effectués sur ce modèle (voir la communication [1], et la section 5.5.3 du livre [3]), mais uniquement dans le cas statique (pas d'évolution des forces extérieures, et donc pas de représentation du caractère irréversible de l'endommagement). Rappelons les principales difficultés numériques liées à la modélisation de l'endommagement. Tout d'abord, bien que le contexte de notre étude soit celui de l'élasticité linéarisée, la détermination simultanée de la répartition des zones endommagées et du champ de déplacements rend ce problème non-linéaire. Par ailleurs, afin que le problème soit bien posé, il faut recourir à l'homogénéisation qui permet de décrire les mélanges fins de matériaux sain et endommagé. Enfin, pour simuler

*Commissariat à l'Energie Atomique, CEA Saclay, 91191 Gif sur Yvette, et Laboratoire d'Analyse Numérique, Université Paris 6, 75252 Paris Cedex 05, Gregoire.Allaire@cea.fr

'Laboratoire d'Analyse Numérique, Université Paris 6, 75252 Paris Cedex 05, aubry@ann.jussieu.fr

${ }^{\ddagger}$ Centre de Mathématiques Appliquées, Ecole Polytechnique 91128 Palaiseau Cedex,

Francois.Jouve@polytechnique.fr 
l'évolution de l'endommmagement il est nécessaire de prendre en compte l'irréversibilité de celui-ci, c'est-à-dire de forcer l'endommagement à être croissant dans le temps.

De nombreuses expériences numériques ont déjà eu lieu pour simuler la propagation de l'endommagement selon divers modèles. Sans prétendre faire une comparaison exhaustive avec celles-ci, disons simplement que notre approche est stable par raffinement du maillage, et ne présente pas de sensibilité par rapport au schéma numérique utilisé. Le principal défaut du modèle est qu'il ne s'applique qu'aux équations de l'élasticité linéarisée, ce qui l'empêche de tenir compte des effets non-linéaires de l'endommagement qui est plus important dans les zones chargées en traction plutôt qu'en compression. Un essai purement numérique est proposé pour y remédier.

Le plan de l'article est le suivant: on commence par rappeler le modèle d'endommagement de Francfort-Marigo, puis on décrit la méthode numérique utilisée ici, enfin on montre sur des exemples son efficacité et ses limites.

\section{Le problème d'évolution de l'endommagement}

Cette section présente le modèle mathématique d'endommagement développé par Francfort et Marigo [4] et les principaux résultats concernant ce modèle. Il s'agit d'un modèle quasi-statique, c'est-à-dire que le temps est discrétisé : on note $\left(t^{i}\right)_{i \in \mathcal{I}}$ la suite croissante des temps discrets.

On considère un domaine $\Omega \subset \mathbb{R}^{2}$ qui au temps initial $t^{0}=0$ est occupé par un matériau sain, élastique, homogène, de loi de Hooke $A_{0}$. A chaque temps $t^{i}$, le corps $\Omega$ est soumis à un chargement surfacique $f^{i}$ qui a pour effet de l'endommager partiellement. Le matériau endommagé est modélisé par un matériau élastique, homogène, de loi de Hooke $A_{1}$, qu'on suppose moins rigide que le matériau sain. Ces deux lois de Hooke sont ordonnées au sens suivant

$$
A_{0} \xi: \xi \geq A_{1} \xi: \xi \geq 0, \forall \xi \text { matrice symétrique. }
$$

En chaque point de $\Omega$, le matériau s'endommage si le champ de contraintes $e(u)$ vérifie

$$
\frac{1}{2}\left(A_{0}-A_{1}\right) e(u): e(u) \geq \lambda,
$$

où $\lambda>0$ est le taux volumique d'énergie libérée par l'endommagement. Ce critère énergétique d'endommagement est connu sous le nom de critère de Griffith. Bien entendu, pour savoir si un point de $\Omega$ est endommagé il faut connaitre au préalable le champ de contraintes qui, à son tour, requiert la connaissance de la loi de Hooke, saine ou endommagée, en chaque point. Il s'agit donc d'un problème couplé non-linéaire, même si les équations sont celles de l'élasticité linéarisée.

Le caractère irréversible de l'endommagement est modélisé par le fait que, si au cours d'un pas de temps $t^{i}$ un point du domaine est endommagé, alors il le reste pour tous les pas de temps suivants $t^{k}, k>i$. On note $\chi_{i}(x)$ la fonction caractéristique, au pas de temps $t^{i}$, de la zone endommagée. Par convention, $\chi_{0}(x)$ est la fonction identiquement nulle (initialement, il n'y a pas d'endommagement). La formulation originale du problème d'évolution de l'endommagement consiste alors à trouver, en suivant les temps croissants, la fonction caractéristique $\chi_{i}(x)$ qui minimise la fonctionnelle d'énergie suivante

$$
\begin{aligned}
& \text { inf } \chi(x)=0,1 \quad\left[\operatorname { m i n } _ { u \in H ^ { 1 } ( \Omega ) ^ { 2 } } \left(\frac { 1 } { 2 } \int _ { \Omega } \left(\chi(x) A_{1}+\right.\right.\right. \\
& \chi(x) \geq \chi_{i-1}(x) \\
& \left.\left.\left.(1-\chi(x)) A_{0}\right) e(u): e(u)-\int_{\partial \Omega} f^{i} \cdot u\right)+\int_{\Omega} \lambda \chi(x)\right]
\end{aligned}
$$

Francfort et Marigo ont montré que ce premier modèle est mal posé, car il n'admet pas, en général, de minimisateur. En effet, des mélanges très fins de matériaux sain et endommagé permettent de diminuer la valeur de la fonctionnelle d'énergie. Par conséquent, il 
est nécessaire de relaxer ce premier modèle en autorisant l'apparition de matériaux composites obtenus par homogénéisation de tels mélanges. Francfort et Marigo ont alors calculé sa relaxation qui conduit à un deuxième modèle d'endommagement, dit stable car il admet toujours une solution. Cette formulation relaxée consiste à trouver la densité de matériau endommagé $0 \leq \theta^{i}(x) \leq 1$, ainsi que la microstructure du mélange caractérisée par une loi de Hooke homogénéisée $A^{i}(x)$, qui minimisent

$$
\min _{\substack{\theta(x) \geq \theta_{i-1}(x) \\ A(x) \in G_{\theta(x)}}}\left[\min _{u \in H^{1}(\Omega)^{2}}\left(\frac{1}{2} \int_{\Omega} A(x) e(u): e(u)-\int_{\partial \Omega} f^{i} \cdot u\right)+\int_{\Omega} \lambda \theta(x)\right]
$$

où $G_{\theta}$ est l'ensemble de tous les matériaux composites obtenus par mélange de $A_{0}$ et $A_{1}$ en proportion $1-\theta$ et $\theta$. Les bornes sur $G_{\theta}$ de l'énergie élastique sont atteintes pour une classe particulière de composites : on peut restreindre cet ensemble $G_{\theta}$ à celui des matériaux composites de la classe des laminés séquentiels de rang 2 . En chaque point $x \in \Omega$, suivant les valeurs de $e(u)(x)$, le tenseur optimal $A(x)$ est un laminé de rang un ou de rang deux connu explicitement (voir [4]).

Le caractère irréversible de l'endommagement se traduit par le fait que la densité $\theta^{i}(x)$, en plus d'appartenir à l'intervalle [0,1], est astreinte à être croissante, i.e. $\theta^{i}(x) \geq \theta^{i-1}(x)$. Un défaut subtil de ce modèle relaxé est que la microstructure n'est pas irréversible (les directions de lamination peuvent varier d'un pas de temps à l'autre).

\section{La méthode numérique}

Le mécanisme d'endommagement mis en oeuvre dans le modèle de Francfort et Marigo est très semblable à celui de l'optimisation de formes par homogénéisation (voir, par exemple, [2], [3]). Il mène à la minimisation d'une fonctionnelle analogue et à des algorithmes numériques voisins. Dans les deux cas, le principe est de trouver un mélange de $A_{0}$ et $A_{1}$ qui soit optimal pour un critère énergétique simple. Ici, la minimisation de (1) conduit à obtenir le mélange le plus faible possible, tandis que pour l'optimisation de formes il faut trouver le plus rigide possible. Le code de calcul que nous avons utilisé est donc analogue à celui décrit en [2] pour résoudre un problème d'optimisation de formes.

L'algorithme que nous allons décrire maintenant est une version de l'algorithme des directions alternées qui a déjà prouvé son efficacité en optimisation de formes (voir [1], [2] pour les détails).

Etant donné un domaine $\Omega$, qui au temps initial $t^{0}=0$ est entièrement occupé par le matériau sain, et une suite de temps discrets croissants $0<t^{1}<t^{2}<\ldots<t^{p}$ et de chargements $\left(f^{i}\right)_{1 \leq i \leq p}$, on calcule, dans l'ordre des temps croissants, pour chaque pas de temps $t^{i}$, de la façon suivante:

1. On initialise le calcul avec $\left(\theta_{0}^{i}, A_{0}^{i}\right)=\left(\theta^{i-1}, A^{i-1}\right)$.

2. Jusqu'à convergence on itère en calculant successivement:

(a) le tenseur de déformations $e_{n}^{i}$ avec les paramètres fixés $\left(\theta_{n}^{i}, A^{i}{ }_{n}\right)$,

(b) les paramètres d'endommagement $\quad\left(\theta_{n+1}^{i}, A_{n+1}^{i}\right), \quad$ avec la contrainte $\theta_{n+1}^{i} \in\left[\theta^{i-1}, 1\right]$, pour les déformations $e_{n}^{i}$.

Le critère d'arrêt des itérations est atteint lorsque la quantité suivante est suffisamment petite

$$
\max \left(\max _{j}\left|\left(\theta_{n+1}^{i}\right)_{j}-\left(\theta_{n}^{i}\right)_{j}\right|, 1-\frac{\int_{\Omega} A_{n+1}^{i} e_{n+1}^{i}: e_{n+1}^{i}+\int_{\Omega} \lambda \theta_{n+1}^{i}}{\int_{\Omega} A_{n}^{i} e_{n}^{i}: e_{n}^{i}+\int_{\Omega} \lambda \theta_{n}^{i}}\right)
$$

Dans les essais numériques, le critère d'arrêt est fixé à $10^{-5}$. 
Les différents problèmes d'élasticité linéarisée pour calculer les champs de déformations $e_{n}^{i}$ sont résolus par la méthode des éléments finis avec des éléments $\mathcal{Q} 1$ pour les déplacements et une valeur au centre de chaque élément pour les paramètres d'endommagement. La loi de Hooke du composite optimal $A_{n}^{i}$ est programmée explicitement grâce aux formules déjà évoquées en première partie. Enfin, on détermine la densité optimale $\theta_{n}^{i}$ par minimisation sur l'intervalle $\left[\theta^{i-1}, 1\right]$ de la fonction $\psi(\theta)=A(\theta, e) e: e+\lambda \theta$ qui est convexe en $\theta$.

\section{Les résultats numériques}

Les cas tests que nous présentons ont été réalisés sur une moitié du domaine puis symétrisés à l'aide d'un logiciel graphique.

\subsection{Evolution de l'endommagement autour d'une fissure}

Cette section est consacrée à l'étude de la propagation progressive de l'endommagement lorsque le domaine $\Omega$ est une éprouvette sur laquelle on a créé une fissure initiale. Le chargement est celui de la figure 1. L'espace de travail $\Omega$ est discrétisé avec 2 x 20000 éléments et les données sont les suivantes:

$$
E_{0}=5.5, \quad \nu_{0}=0.25, \quad E_{1}=0.2, \quad \nu_{1}=0, \quad \lambda=10
$$

où les $E_{i}$ sont les modules de Young et les $\nu_{i}$ sont les coefficients de Poisson du matériau $i$, $i=0,1$. La figure 1 montre l'évolution de l'endommagement le long de la fissure lorsqu'on lui applique une force d'intensité croissante en temps.

Lorsque le coefficient de Poisson du matériau endommagé est non nul, la zone endommagée évolue de façon plus isotrope en s'éloignant de l'axe de symétrie du domaine. L'observation du champ de déplacements montre que ce phénomène est principalement dû à des torsions du domaine n'intervenant pas lorque $\nu_{1}$ est nul. Plus $\nu_{1}$ s'approche de $\frac{1}{2}$, plus la zone endommagée se détache de l'axe.

$\mathrm{Si}$ on considère maintenant l'endommagement obtenu lorsque les déplacements sont imposés sur le bord du domaine et non plus les forces, on remarque que des zones d'endommagement supplémentaires apparaissent, elles montrent les contraintes latérales exercées sur le domaine pour respecter le déplacement imposé au bord.

\subsection{Comparaison entre les cas stationnaire et quasi-stationnaire}

Pour le même chargement que précédemment (cf. figure 1) avec les mêmes données initiales, on applique maintenant directement l'intensité maximale du chargement (correspondant au dernier pas de temps). L'endommagement que l'on obtient alors est le même que précédemment. Dans ce cas, l'algorithme est donc insensible à l'historique du chargement et de l'évolution de l'endommagement.

Pour faire apparaitre le caractère irréversible de l'endommagement et comparer les cas stationnaire et quasi-stationnaire, on fait le cas test suivant: on alterne les deux chargements représentés sur la figure 2, en commençant par le premier et en augmentant la force à chaque pas de temps, l'endommagement obtenu est représenté sur la figure 3 pour un maillage constitué de 2 x 5000 éléments. On le compare alors au cas test qui consiste à prendre uniquement le premier chargement de la figure 2 pour une force d'intensité égale à celle du dernier pas de temps précédent ( $c$ f. figure 4). On observe alors la permanence des zones endommagées aux instants suivants. 


\subsection{Questions numériques}

La convergence de l'algorithme des directions alternées est liée à la décroissance de la fonction objectif donnée par $-\frac{1}{2} \int_{\Omega} A_{n} e_{n}(u): e_{n}(u)+\int_{\Omega} \lambda \theta_{n}$ à chaque itération $n$ de l'algorithme. Dans les cas tests précédents, la convergence est très lente puisqu'il faut à peu près 300 itérations pour converger vers la solution mais la décroissance de la fonction objectif est monotone.

Cependant, lorsque le rapport entre les propriétés des matériaux sain et endommagé est élevé (par exemple, $E_{0}=21.8, \nu_{0}=0.28$ et $E_{1}=0.037, \nu_{1}=0.485$ ), la valeur de la fonction objectif oscille d'une itération à l'autre au bout d'un certain nombre d'itérations et entraine la non-convergence de l'algorithme. Ces oscillations sont probablement provoquées par le mauvais conditionnement de la matrice éléments finis.

\subsection{Modification non-linéaire}

En réalité l'endommagement est plus important en traction qu'en compression. Le code ne montre pas cette différence car le modèle Francfort-Marigo a pour cadre l'élasticité linéarisée. Pour tenir compte de cet effet non-linéaire de l'endommagement, on introduit la modification suivante sur le taux volumique $\lambda$ d'énergie libérée par endommagement.

On se donne deux valeurs différentes de ce taux volumique: $\lambda_{c}$ en compression et $\lambda_{t}<\lambda_{c}$ en traction. La différence entre la traction et la compression se lit sur les valeurs propres. On pose $\sigma_{i}, i=1,2$ les valeurs propres du tenseur des contraintes. On propose la modification suivante:

Si $\sigma_{i}<0, \forall i=1,2$, il y a compression et on pose $\lambda=\lambda_{c}$

Si $\sigma_{i}>0, \forall i=1,2$, il y a traction et on pose $\lambda=\lambda_{t}$.

Si $\sigma_{i}<0$ et $\sigma_{j}>0 \forall i \neq j$, alors il y a cisaillement et on convient du critère moyenné suivant:

$$
\lambda_{m}=\frac{\lambda_{c}\left|\sigma_{i}\right|+\lambda_{t}\left|\sigma_{j}\right|}{\left|\sigma_{i}\right|+\left|\sigma_{j}\right|}
$$

En effet, plus $\lambda$ est élevé, plus l'endommagement est faible. On veut moins endommager en compression qu'en traction, $\lambda_{c}$ doit donc être plus grand que $\lambda_{t}$. Cette modification, purement numérique, transforme le taux volumique $\lambda$ en une variable dépendant du champ de contraintes.

Sur les cas tests où nous avons essayé cette modification, nous avons constaté que l'énergie élastique ne cessait de décroitre à chaque itération et que la non linéarité de l'endommagement était plus évidente. Nous avons aussi remarqué que l'algorithme présentait des instabilités lorsque $\lambda_{c}$ était trop grand par rapport à $\lambda_{t}$. La figure 6 montre cette modification dans le cas d'une poutre en flexion pour $\lambda_{c}=3 \lambda$ discrétisé avec 2 x 2100 éléments.

\section{Références}

[1] G. Allaire \& G. FrancFort, Numerical aspects of damage, SIAM Conference on Mathematical Aspects of Material Sciences, Pittsburgh, (1994).

[2] G. Allaire, E. Bonnetier, G. Francfort \& F. Jouve, Shape optimisation by the homogenization method, Num. Math., 76, (1997), pp. 27-68.

[3] M. Bendsoe, Optimization of structural topology, shape, and material, Springer Verlag, (1995).

[4] G. Francfort \& J.J Marigo, Stable damage evolution in a brittle continuous medium, European Journal Mech. A Solids, 12, (1993), pp. 149-189. 
[5] J. Lemaitre \& J.-L. Chaboche, Aspect phénoménologique de la rupture par endommagement, J. Mécanique Appliquée, 2, (1978), pp. 317-365.
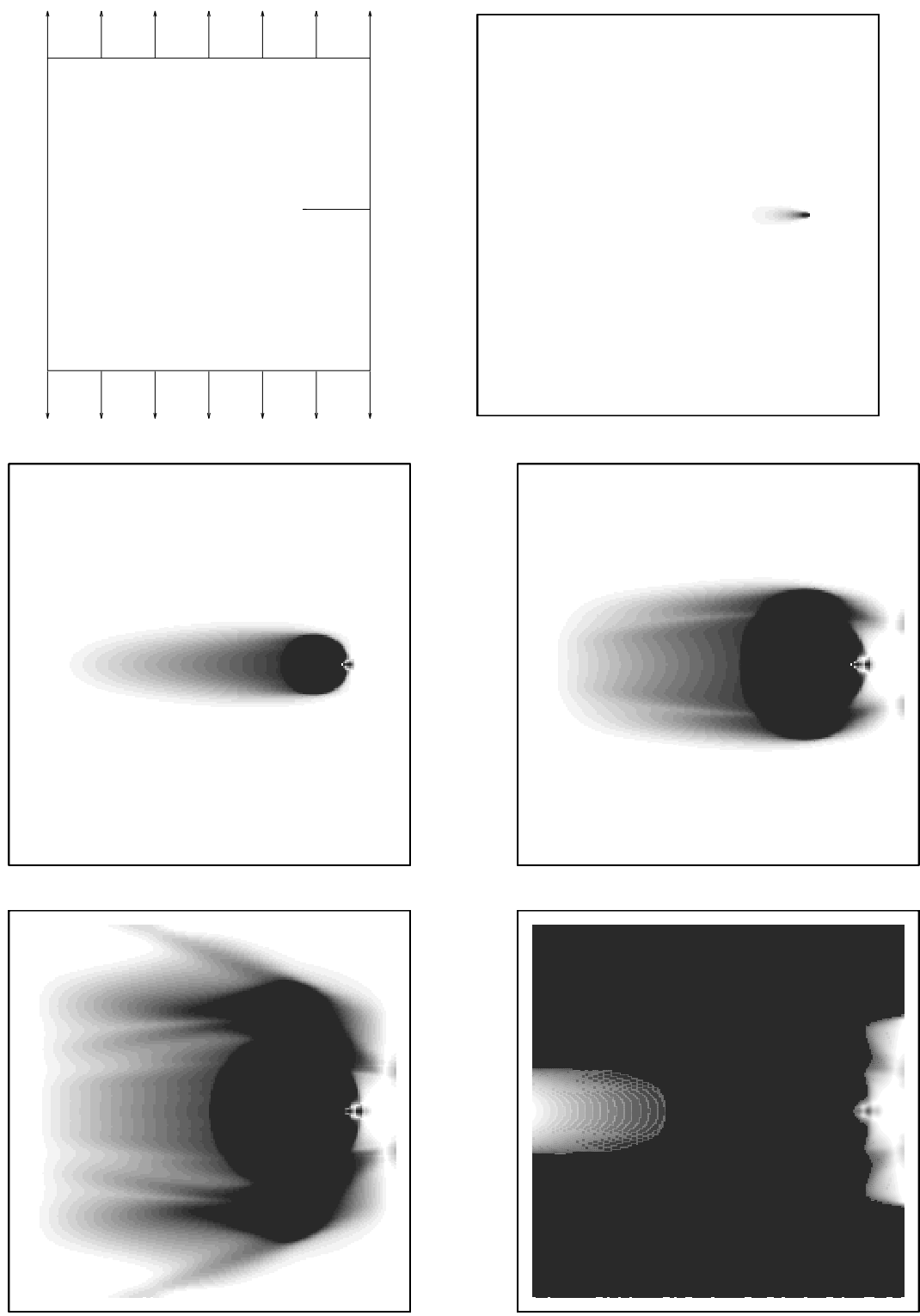

Fig. 1 - Endommagement autour d'une fissure pour 5 pas de temps lorsque l'intensité de la force est augmentée progressivement à chaque pas de temps 

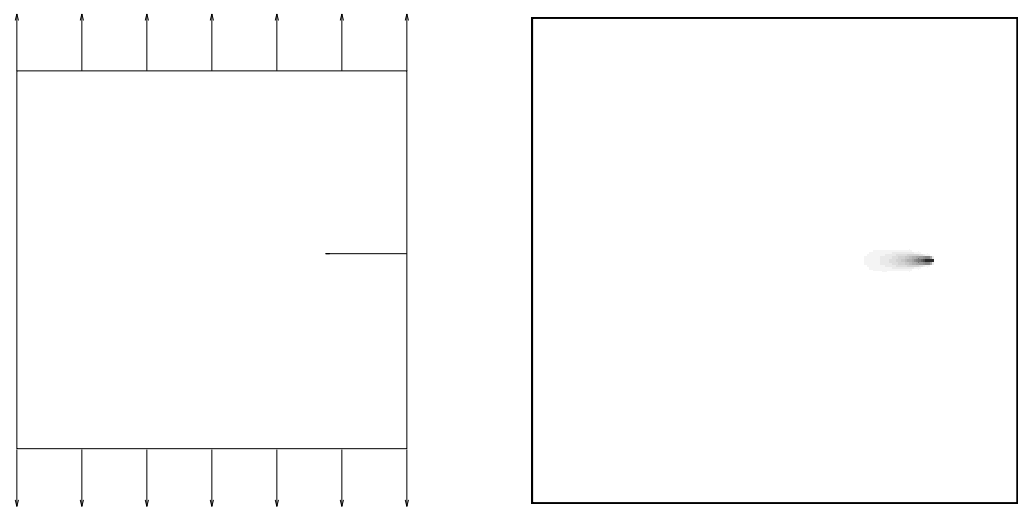

Fig. 2 - Les deux types de chargement que l'on alterne à chaque pas de temps
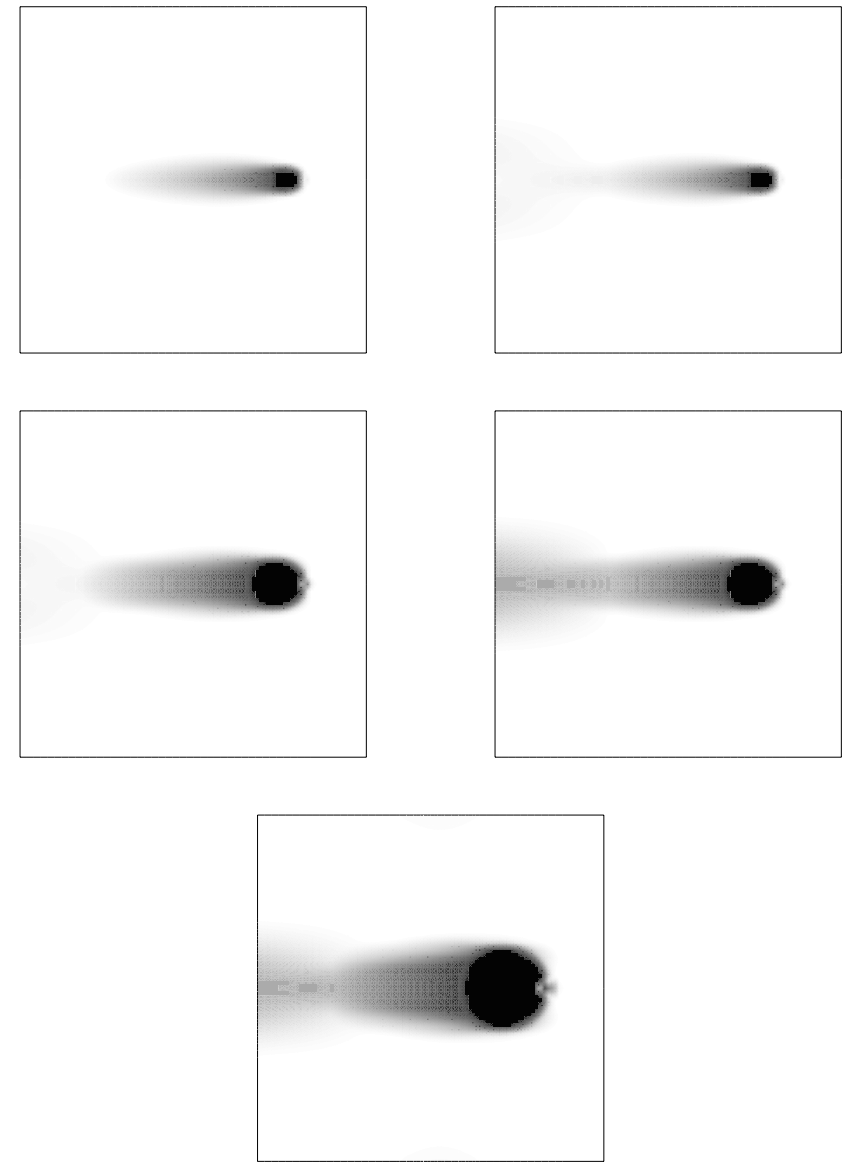

Fig. 3 - Eprouvette pré-fissurée dans le cas quasi-stationnaire, la force est augmentée à chaque pas de temps et le chargement diffère d'un pas de temps à l'autre 


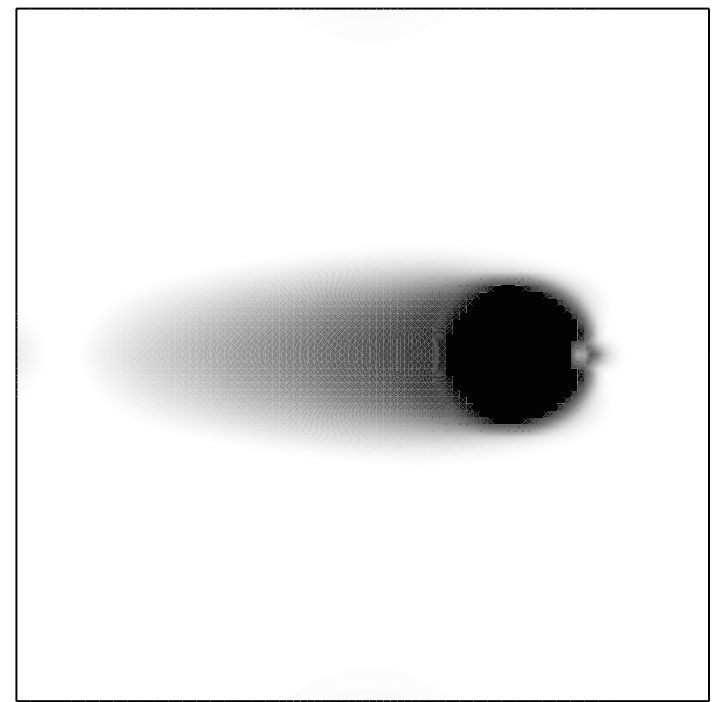

Fig. 4 - Eprouvette pré-fissurée dans le cas stationnaire

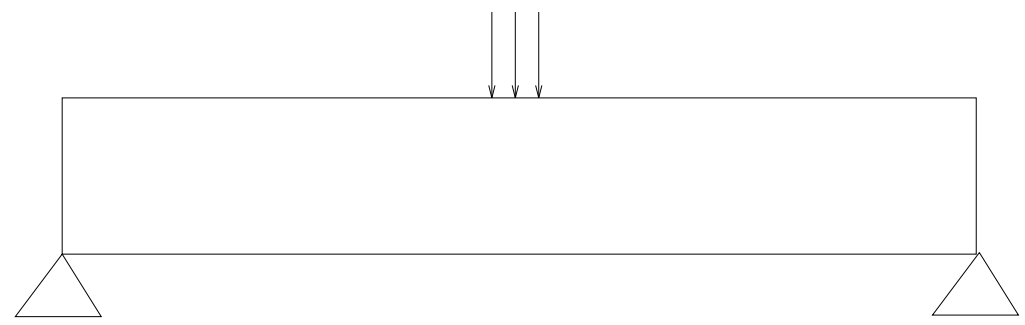

FIG. 5 - Le chargement

ESAIM: ProC., VOL. 3, 1998, 1-9 

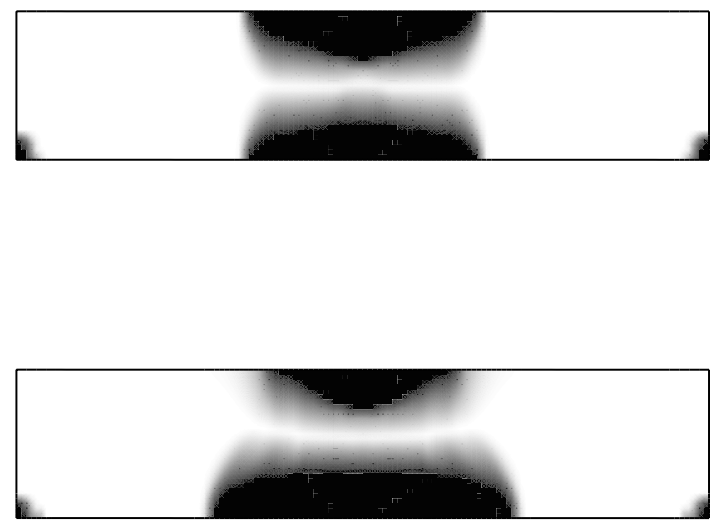

FIg. 6 - Poutre en flexion. Les cas linéaire (en haut) et non linéaire (en bas) avec respectivement $\lambda=20$ et $\lambda=10$ 